\title{
Diabetes Mellitus Is Associated with a Lower Risk of Gout: A Meta-Analysis of Observational Studies
}

\author{
Xiaoli Li $\mathbb{D},,^{1,2,3}$ Lianju Li $\mathbb{D},{ }^{1}$ Yuling Xing $\mathbb{D},{ }^{2,3}$ Tiantian Cheng $\mathbb{D},^{3}$ Shaohui Ren $\mathbb{D}^{4}$ \\ and Huijuan Ma iD $^{2,3,5}$ \\ ${ }^{1}$ Department of Rheumatology, Xingtai People's Hospital Affiliated to Hebei Medical University, Xingtai 054001, China \\ ${ }^{2}$ Department of Internal Medicine, Hebei Medical University, Shijiazhuang 050017, China \\ ${ }^{3}$ Department of Endocrinology, Hebei General Hospital, Shijiazhuang 050051, China \\ ${ }^{4}$ Department of Medicine, Xingtai People's Hospital Affiliated to Hebei Medical University, Xingtai 054001, China \\ ${ }^{5}$ Hebei Key Laboratory of Metabolic Diseases, Hebei General Hospital, Shijiazhuang 050051, China
}

Correspondence should be addressed to Huijuan Ma; huijuanma76@163.com

Received 2 May 2020; Revised 22 June 2020; Accepted 23 June 2020; Published 11 July 2020

Academic Editor: Eric Hajduch

Copyright ( 2020 Xiaoli Li et al. This is an open access article distributed under the Creative Commons Attribution License, which permits unrestricted use, distribution, and reproduction in any medium, provided the original work is properly cited.

\begin{abstract}
Aims. Although several epidemiological studies have investigated the relationship between diabetes mellitus (DM) and the risk of gout, the results are inconsistent. Therefore, we systematically retrospected available observational studies to clarify the impact of DM on the risk of gout. Methods. Embase, PubMed, Cochrane Library, Scopus, Web of Science, and China National Knowledge Infrastructure were searched for relevant articles from inception to 2 March 2020. The quality of the included studies was assessed using the Newcastle-Ottawa Quality Assessment Scale. The multivariate adjusted relative risks (aRR) and corresponding 95\% confidence intervals (CI) were pooled based on a random-effect model. Cochran's $Q$ test and $I^{2}$ were used to evaluate heterogeneity. Results. Five studies involving 863,755 participants were included in our meta-analysis. DM was associated with a lower risk of gout (aRR: $0.66 ; 95 \%$ CI: 0.59 to 0.73 ) but had a high heterogeneity $\left(I^{2}=89.2 \%\right)$. Metaregression analysis revealed that the types of DM were the source of heterogeneity. Subgroup analysis by types of DM showed that the risk of gout was significantly lower in type $1 \mathrm{DM}$ (T1DM) (aRR: 0.42 ; 95\% CI: 0.28 to 0.63 ) than in type 2 DM (T2DM) (aRR: 0.72 ; 95\% CI: 0.70 to 0.74$)$. Furthermore, when stratified according to gender in DM, sex-specific association was found. The inverse association was observed in males only (aRR: 0.57; 95\% CI: 0.43 to 0.77) and not in females (aRR: 0.96; 95\% CI: 0.87 to 1.05 ). Further stratified based on glycated hemoglobin (HbA1c) levels in DM, raised A1C levels were associated with a reduced risk of gout in patients with DM. Conclusions. This meta-analysis indicated that DM was related to a lower risk of gout, and the protective effect of DM on the risk of gout was stronger in males, T1DM, or DM with high HbA1c levels. However, more prospective cohort studies are required to confirm these results.
\end{abstract}

\section{Introduction}

Gout is a crystal-associated arthropathy characterized by the deposition of monosodium urate (MSU), which is directly related to hyperuricemia caused by disorders of purine metabolism and/or decreased uric acid excretion. The prevalence of gout accounts for approximately $5 \%$ of the middleaged and elderly global population, and the incidence of gout has increased steadily in recent years $[1,2]$. Individuals with T2DM generally have a higher prevalence of high blood pres- sure [3], obesity, and decreased kidney function [4]. These comorbid conditions are also risk factors of gout. Both DM and gout are related to a high risk of cardiovascular events, kidney failure, and mortality [4-7]. Therefore, the relationship between DM and gout has attracted great attention.

Several prospective studies found that gout was positively associated with the risk of DM [8-11]. Similarly, a metaanalysis of 11 cohort studies with 42,834 participants reported a positive correlation between serum uric acid level and the risk of DM [12]. However, the impact of DM on the 
risk of gout was inconsistent. Several small cross-sectional studies showed that DM was associated with a higher risk of gout [13-16], whereas a prospective cohort study [11] and a case-control study [17] suggested that DM was negatively correlated with the risk of gout, and no association was found in another prospective cohort study [18]. Therefore, we retrospected available observational studies to clarify the impact of DM on the risk of gout.

\section{Methods}

2.1. Search Strategy. This meta-analysis was registered in PROSPERO with the registration number CRD42020159645. Six databases including Embase, PubMed, Cochrane Library, Scopus, Web of Science, and China National Knowledge Infrastructure (CNKI) were searched for relevant articles by two authors (X-L L and Y-L X) independently from inception to 2 March 2020. The search strategy was the combination of the MeSH terms and entry terms for "Gout or Gout arthritis" and "DM or T1DM or T2DM." Meanwhile, the searched studies were limited to human beings and there was no restriction in languages. Taking PubMed and Embase databases for example, the details of the retrieval process are listed in supplementary material Excel S1-S2.

2.2. Eligibility Criteria. The purpose of this study was to explore the impact of DM on the risk of gout. Our eligibility criteria are as follows: (1) Studies should have an observational design and should investigate the relationship between DM and the risk of gout. (2) Studies should diagnose DM prior to the diagnosis of gout. (3) Outcomes should be presented as the multivariate adjusted relative risk (RR), odds ratio (OR), or hazard ratio (HR) with corresponding $95 \%$ CI. (4) Studies should involve subjects without DM or gout as the corresponding control group.

2.3. Study Selection and Data Extraction. The screening of articles was independently performed by three authors (XL L, L-J L, and T-T C). For inconsistent results, we would discuss them together or solicit the final judgment of the senior researcher (H-J M). Standardized data sheets were used by the abovementioned investigators to independently collect data. The following information was extracted: first author, year of publication, study design, data source, study period, definition of cases and controls, ascertainment of $\mathrm{DM}$ /gout, follow-up period, numbers of cases and controls, sex ratio, ages of cases and controls, types of DM, adjustment confounders, and adjusted OR/RR/HR (95\% CI). All entries were confirmed by two of the authors mentioned above and checked at least twice to ensure accuracy and completeness.

2.4. Study Quality. The quality of the included studies was assessed using the Newcastle-Ottawa Quality Assessment Scale (NOS) [19]. As a quality assessment tool, NOS evaluated the quality of a study through three aspects: 4 stars for selection, 2 stars for comparability, and 3 stars for exposure/results, with a total of 9 stars for case-control studies and cohort studies. A score of $0-5$ was regarded as low quality, while a score that reached six or more stars was considered to be high quality [20]. To ensure accuracy, the process of quality assessment was conducted by two authors independently and supervised by the senior researcher (H-J M).

2.5. Statistical Analysis. As the incidence of gout was relatively low $(<5 \%)[1]$, the OR of a case-control study or the HR of a cohort study was used as an estimate for RR to compute the pooled RRs [21]. The multivariate adjusted relative risks (aRR) and corresponding 95\% CI reported in the studies were used to produce forest plots in our meta-analysis. Heterogeneity was evaluated by Cochran's $Q$ test and $I^{2}$. The degree of heterogeneity was judged as follows: $0 \%<I^{2}$ $\leq 25 \%$ represented insignificant heterogeneity, $25 \%<I^{2} \leq$ $50 \%$ indicated low heterogeneity, $50 \%<I^{2} \leq 75 \%$ showed moderate heterogeneity, and $I^{2}>75 \%$ represented high heterogeneity [22]. If $I^{2}<50 \%$, the heterogeneity between groups was low and a fixed-effect model was used. Whereas, for $I^{2}>50 \%$, the heterogeneity was obvious and a randomeffect model was used. Metaregression analysis and subgroup analysis were performed to explore the source of heterogeneity. To identify sources of heterogeneity and assess the robustness of the results, sensitivity analysis was conducted by removing each study individually and calculating a pooled effect estimate for the remaining studies to assess whether a single study affected the results. All statistical analyses were conducted with STATA 14.0 software.

\section{Results}

3.1. Literature Search Results. By searching six databases, 3254 potentially relevant articles were identified (787 from Embase, 503 from PubMed, 13 from Cochrane Library, 971 from Scopus, 355 from Web of Science, and 625 from CNKI). After checking the records and removing duplicates, 2423 articles were screened by titles and abstracts. 2392 articles were removed due to irrelevant studies, leaving 31 articles for full-text review. 26 of the 31 articles were rejected for the following reasons: the effect of gout/hyperuricemia on the risk of DM $(n=12)$, not event as outcome $(n=3)$, subject not relevant $(n=3)$, studies without control group $(n=5)$, study failure to prove the diagnosis of DM prior to gout $(n=1)$, republished study $(n=1)$, and incomplete data $(n=1)$. Finally, five studies $[11,17,18,23,24]$ met the inclusion criteria and were included in this meta-analysis. The screening process is shown in Figure 1.

3.2. Characteristics of the Included Studies. Three cohort studies [11, 18, 23] and two case-control studies [17, 24] involving 863,755 participants were included in this metaanalysis. The included studies were published from 2010 to 2016. Of the three cohort studies, two studies $[11,18]$ explored the relationship between DM and the risk of gout, while another study [23] showed the impact of T2DM on the risk of gout. Two case-control studies [17, 24] revealed the impact of T1DM and T2DM on the risk of gout, respectively. Four studies $[11,17,18,23]$ discussed the impact of gender differences of patients with DM on the risk of gout. All studies were based on large databases, and the diagnosis of DM or gout was mainly based on diagnostic codes, self- 


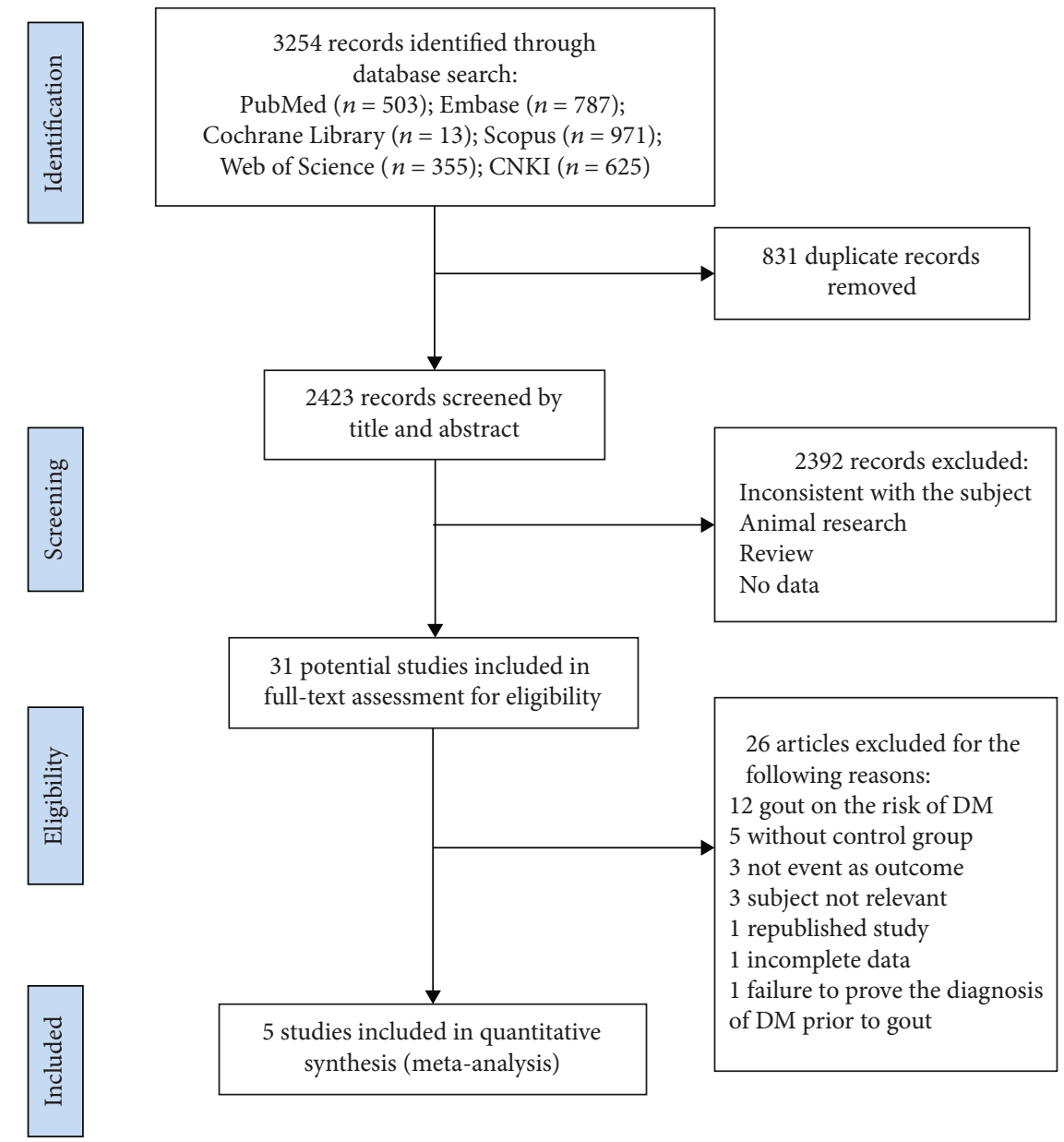

FIGURE 1: Flow chart of literature selection. CNKI: China National Knowledge Infrastructure; DM: diabetes mellitus.

report, prescriptions for drug use, or laboratory findings. The quality of the included studies was evaluated according to NOS, and the NOS scores ranged from 6 to 8 . The detailed characteristics of the included studies are illustrated in Tables 1 and 2 .

3.3. Overall Meta-Analysis and Sensitivity Analysis. Given that two case-control studies $[17,24]$ discussed the impact of T1DM and T2DM on the risk of gout, respectively, therefore, each of the two studies combined the effect estimate according to two studies. Finally, a pooled effect estimate to assess the impact of DM on the risk of gout was calculated from seven studies with a total of 863,755 participants. Compared with the control groups, the pooled aRR of gout in patients with DM was 0.66 (95\% CI: 0.59 to 0.73 ), but the heterogeneity was high $\left(I^{2}=89.2 \%\right)$, as shown in Figure 2. To identify sources of heterogeneity and assess the robustness of results, sensitivity analysis was performed by removing each study individually, and the estimated aRRs in the sensitivity analysis ranged from 0.64 (95\% CI: 0.54 to 0.75 ) to 0.70 ( $95 \%$ CI: 0.64 to 0.77 ) (Table S1). Deletion of any single study did not change the overall statistical significance, showing that the results were steady and reliable in statistics. As less than 10 studies were included, no funnel plot was produced to assess publication bias.
3.4. Subgroup Analysis and Metaregression Analysis. To further explore sources of heterogeneity, a metaregression analysis and a subgroup analysis were performed according to geographical location, study design, and types of DM. In the subgroup analysis, the majority of strata showed inverse association between DM and the risk of gout. However, except for types of DM, there was no significant statistical significance between subgroups with a metaregression analysis, indicating that types of DM contributed the most to the heterogeneity (Table 3).

Subgroup analysis by types of DM (two studies in DM $[11,18]$, three studies in T2DM [17, 23, 24], and two studies in T1DM $[17,24]$ ) showed that heterogeneity had a significant decrease in the DM subgroup $\left(I^{2}=22.4 \%\right)$ and the T2DM subgroup $\left(I^{2}=0 \%\right)$ but was still high in the T1DM subgroup $\left(I^{2}=81.5 \%\right)$. The risk of gout was significantly lower in T1DM than in T2DM; the pooled aRR in T1DM was 0.42 (95\% CI: 0.28 to 0.63 ) and the aRR in T2DM was 0.72 (95\% CI: 0.70 to 0.74 ), while no association was found in the DM subgroup (aRR: 0.84; 95\% CI: 0.68 to 1.05 ), as shown in Figure 3.

3.5. Sex-Specific Analysis of DM and the Risk of Gout. Considering the gender difference in the incidence of gout, further sex-stratified analysis was discussed between DM and the 


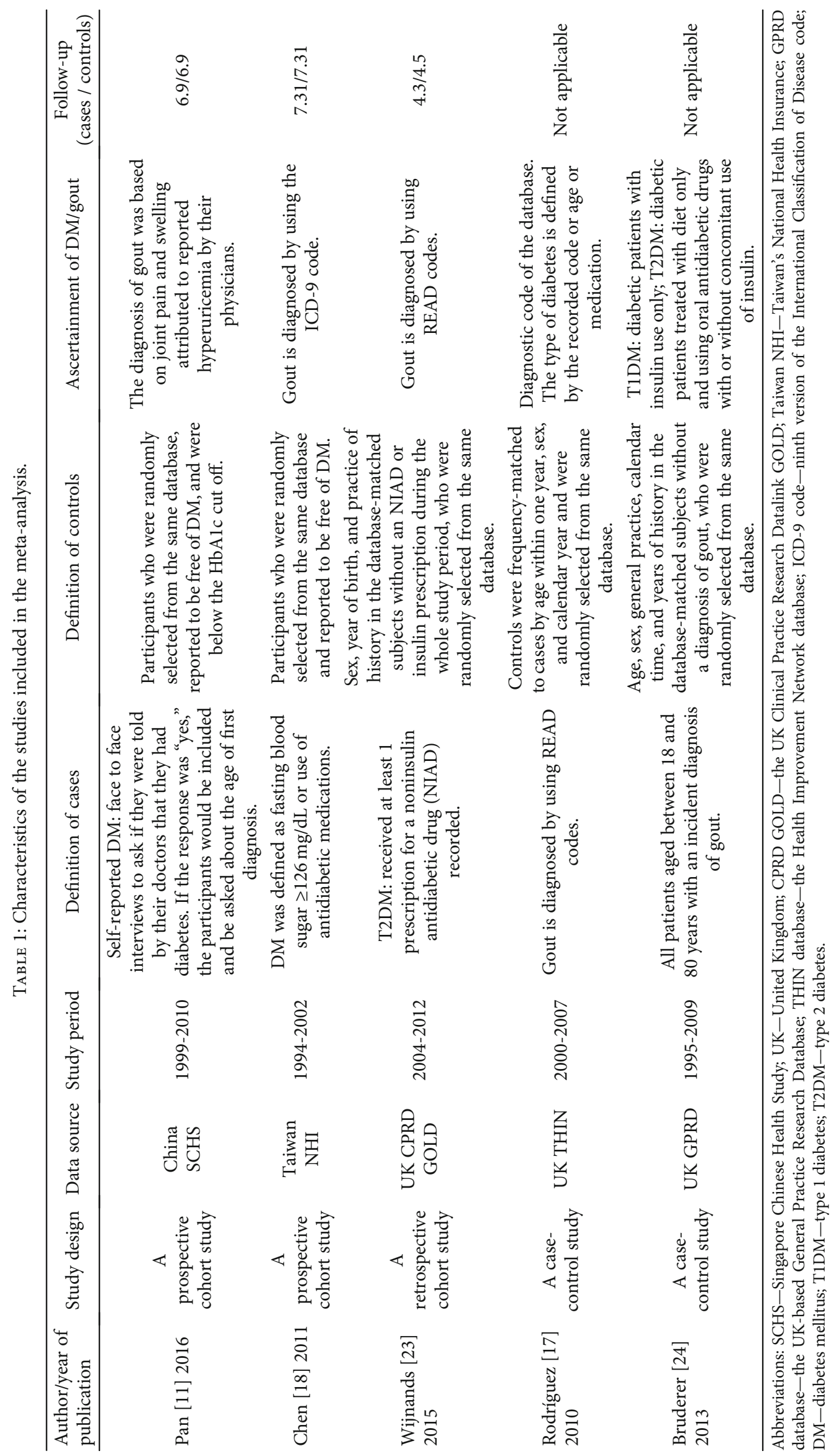




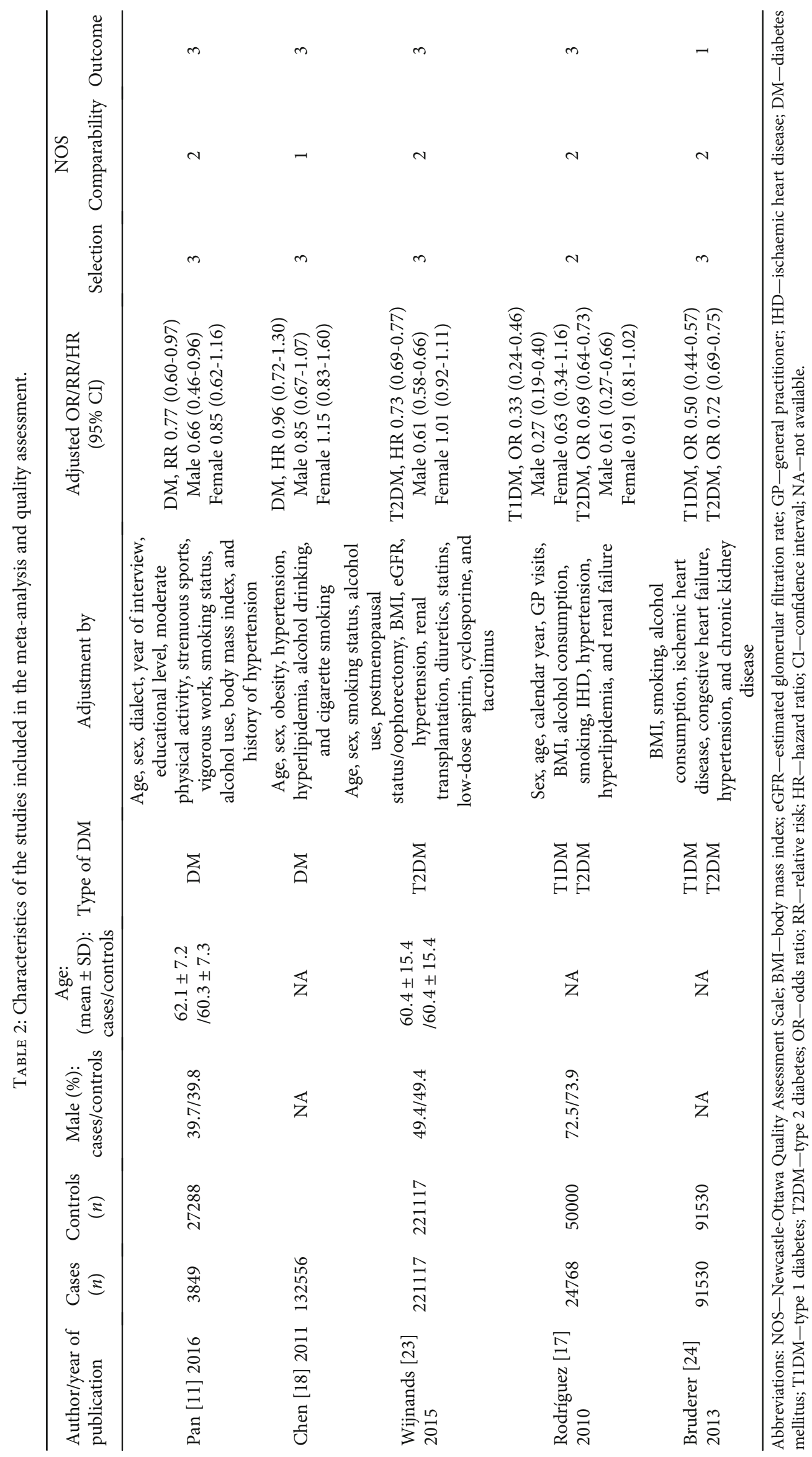




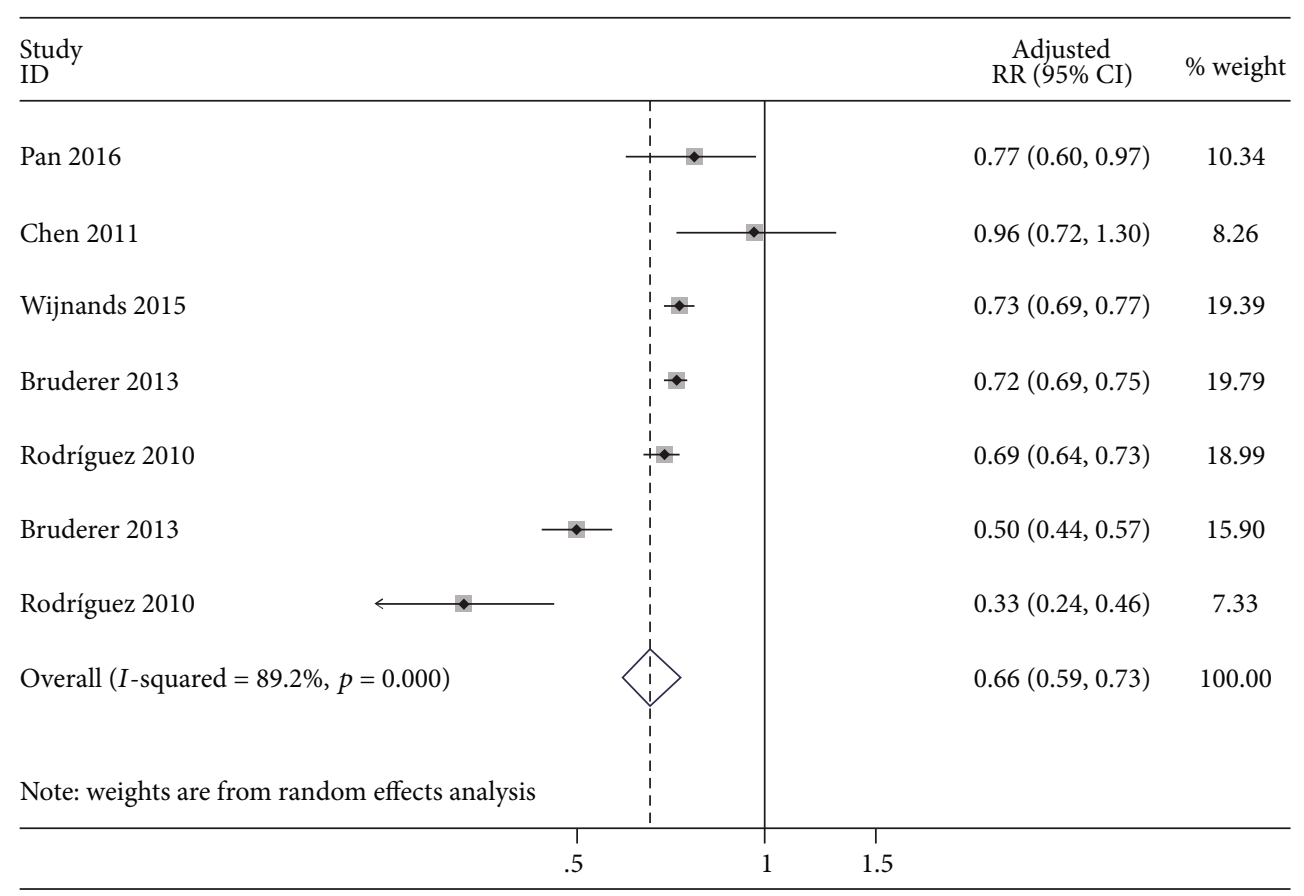

FIGURE 2: Forest plot of the risk of gout in patients with DM compared with controls. DM: diabetes mellitus; RR: relative risk; CI: confidence interval.

TABLE 3: Stratified meta-analysis and metaregression of the association of DM and the risk of gout.

\begin{tabular}{|c|c|c|c|c|c|c|c|}
\hline Covariates & No. of study & RR (95\% CI) & $I^{2}(\%)$ & $\mathrm{Ph}^{*}$ & $\mathrm{Tau}^{2}$ & $\begin{array}{c}\text { Metaregression } \\
\text { Adj }-R^{2}(\%)\end{array}$ & $\mathrm{Ph}^{* *}$ \\
\hline Overall & 7 & $0.66(0.59,0.73)$ & 89.2 & $<0.000$ & & & \\
\hline \multicolumn{8}{|l|}{ Subgroup analyses } \\
\hline Types of DM & & & & & 0.000 & 99.6 & 0.01 \\
\hline DM & 2 & $0.84(0.68,1.05)$ & 22.4 & 0.256 & & & \\
\hline T1DM & 2 & $0.42(0.28,0.63)$ & 81.5 & 0.020 & & & \\
\hline $\mathrm{T} 2 \mathrm{DM}$ & 3 & $0.72(0.70,0.74)$ & 0.0 & 0.415 & & & \\
\hline Study design & & & & & 0.01 & 26.2 & 0.149 \\
\hline Cohort study & 3 & $0.77(0.68,0.88)$ & 39.7 & 0.191 & & & \\
\hline Case-control study & 4 & $0.58(0.48,0.69)$ & 93.7 & $<0.000$ & & & \\
\hline Geographical location & & & & & 0.072 & 16.9 & 0.208 \\
\hline Asia & 2 & $0.84(0.68,1.05)$ & 22.4 & 0.256 & & & \\
\hline Europe & 5 & $0.62(0.55,0.70)$ & 92.1 & $<0.000$ & & & \\
\hline
\end{tabular}

Abbreviations: RR-relative risk; CI-confidence interval; $\mathrm{Ph}^{*}-p$ value for heterogeneity within each subgroup; $\mathrm{Ph}^{* *}-p$ value for heterogeneity between subgroups in metaregression analysis.

risk of gout. Four studies [11, 17, 18, 23] including 345,943 men and 334,752 women showed that there were inverse correlations between T2DM and the risk of gout among males; the pooled aRR was 0.57 (95\% CI: 0.43 to 0.77 ), with high heterogeneity $\left(I^{2}=84.8 \%\right)$. However, the risk disappeared in females; the pooled aRR was 0.96 (95\% CI: 0.87 to 1.05), with low heterogeneity $\left(I^{2}=27.1 \%\right)$ (Figure 4$)$.

3.6. HbA1c Levels and the Risk Gout. To evaluate the impact of HbA1c levels on the risk of gout, two studies [23, 24] were included and stratified according to HbAlc levels in DM. Interestingly, we found that HbAlc levels were inversely related to the risk of gout. Compared with HbAlc $<7.0 \%$, the risk of gout was $22 \%$ reduced among those with HbA1c levels of 7.0-7.9\% (aRR: 0.78; 95\% CI: 0.63 to 0.96 ), $33 \%$ reduced among those with HbA1c levels of $8.0-8.9 \%$ (aRR: $0.67 ; 95 \%$ CI: 0.48 to 0.92 ), and even $46 \%$ reduced among those with $\mathrm{HbAlc}>9 \%$ (aRR: 0.54; 95\% CI: 0.41 to 0.70 ); however, there is high heterogeneity (Figure 5). 


\begin{tabular}{|c|c|c|}
\hline $\begin{array}{l}\text { Study } \\
\text { ID }\end{array}$ & $\begin{array}{c}\text { Adjusted } \\
\text { RR ( } 95 \% \text { CI) }\end{array}$ & $\%$ weight \\
\hline \multicolumn{3}{|l|}{$\mathrm{DM}$} \\
\hline Pan 2016 & $0.77(0.60,0.97)$ & 10.34 \\
\hline Chen 2011 & $0.96(0.72,1.30)$ & 8.26 \\
\hline Subtotal $(I$-squared $=22.4 \%, p=0.256)$ & $0.84(0.68,1.05)$ & 18.60 \\
\hline \multicolumn{3}{|l|}{ T2DM } \\
\hline Wijnands 2015 & $0.73(0.69,0.77)$ & 19.39 \\
\hline Bruderer 2013 & $0.72(0.69,0.75)$ & 19.79 \\
\hline Rodriguez 2010 & $0.69(0.64,0.73)$ & 18.99 \\
\hline Subtotal $(I$-squared $=0.0 \%, p=0.415)$ & $0.72(0.70,0.74)$ & 58.17 \\
\hline \multicolumn{3}{|l|}{ T1DM } \\
\hline Bruderer 2013 & $0.50(0.44,0.57)$ & 15.90 \\
\hline Rodríguez 2010 & $0.33(0.24,0.46)$ & 7.33 \\
\hline Subtotal $(I$-squared $=81.5 \%, p=0.020)$ & $0.42(0.28,0.63)$ & 23.23 \\
\hline Overall $(I$-squared $=89.2 \%, p=0.000)$ & $0.66(0.59,0.73)$ & 100.00 \\
\hline \multicolumn{3}{|l|}{ Note: weights are from random effects analysis } \\
\hline $\begin{array}{l}1 \\
.5\end{array}$ & $\begin{array}{c}1.5 \\
1.5\end{array}$ & \\
\hline
\end{tabular}

FIGURE 3: Subgroup analysis of the risk of gout in individuals with DM: grouped by types of DM. DM: diabetes mellitus; T2DM: type 2 diabetes mellitus; T1DM: type 1 diabetes mellitus; RR: relative risk; CI: confidence interval.

\section{Discussion}

The interplay between DM and gout is intricate. On the one hand, DM may be associated with an increased risk of gout, possibly due to DM-related comorbidities such as hypertension, obesity, and metabolic syndrome [3-7]. On the other hand, some pathophysiological mechanisms in DM may have the opposite effect on the risk of gout such as impaired inflammatory response and uricosuric effect of glycosuria $[17,25]$. The results from this meta-analysis showed that patients with DM had a significantly lower risk of developing gout, especially in T1DM. However, contrary to our conclusions, four small cross-sectional studies suggested that DM was associated with an increased risk of gout [13-16], but since their results were not adjusted for the vital confounding factors of the coexistence of gout and DM, this could explain the positive associations. More importantly, three of these studies [13-15] did not have a control group and none of the studies [13-16] demonstrated that DM was diagnosed earlier than gout. Therefore, they did not meet our inclusion criteria and were not included in our meta-analysis. Moreover, a sex-specific association between DM and the risk of gout was found in this meta-analysis. The inverse association was observed in males only, not in females. In addition, increased $\mathrm{HbAlc}$ levels were associated with a reduced risk of gout in patients with DM.

However, high heterogeneity could not be ignored. Metaregression analysis revealed that types of DM were the source of heterogeneity. Although subgroup analysis by types of DM showed that the heterogeneity had a significant decrease in the DM subgroup $\left(I^{2}=22.4 \%\right)$ and the T2DM subgroup $\left(I^{2}=0 \%\right)$, there was still high heterogeneity in the T1DM subgroup $\left(I^{2}=81.5 \%\right)$ (Figure 3 ). So, the possible causes of high heterogeneity in the T1DM subgroup were further explored. This meta-analysis revealed that the inverse association was observed in males only (aRR: 0.57; 95\% CI: 0.43 to 0.77 ) and not in females (aRR: 0.96 ; $95 \% \mathrm{CI}: 0.87$ to 1.05). Therefore, the higher male ratio with DM meant a lower risk of gout. Interestingly, the proportion of males (cases/controls-72.5\%/73.9\%) in the study by Rodriguez et al. [17] was significantly higher than that in other studies (Pan et al.: cases/controls-39.7\%/39.8\%; Wijnands et al.: cases/controls-49.4\%/49.4\%), as shown in Table 2, which might cause the lower risk of gout (aRR: 0.33; 95\% CI: 0.24 to 0.46 ) and high heterogeneity in the T1DM subgroup. Unfortunately, the other study [24] in the T1DM subgroup did not provide the sex ratio of the participants, and even though we exerted every effort to contact the author, no sufficient raw data was obtained. More prospective cohort studies are needed to verify the results and provide more evidence. Another concern with subgroup analysis by types of DM was that the risk disappeared in the DM subgroup. It should be noted that of all the included studies, only the study by Chen et al. [18] found no negative correlation between DM and the risk of gout, finally leading to no statistical significance in the DM subgroup. So, the possible causes 


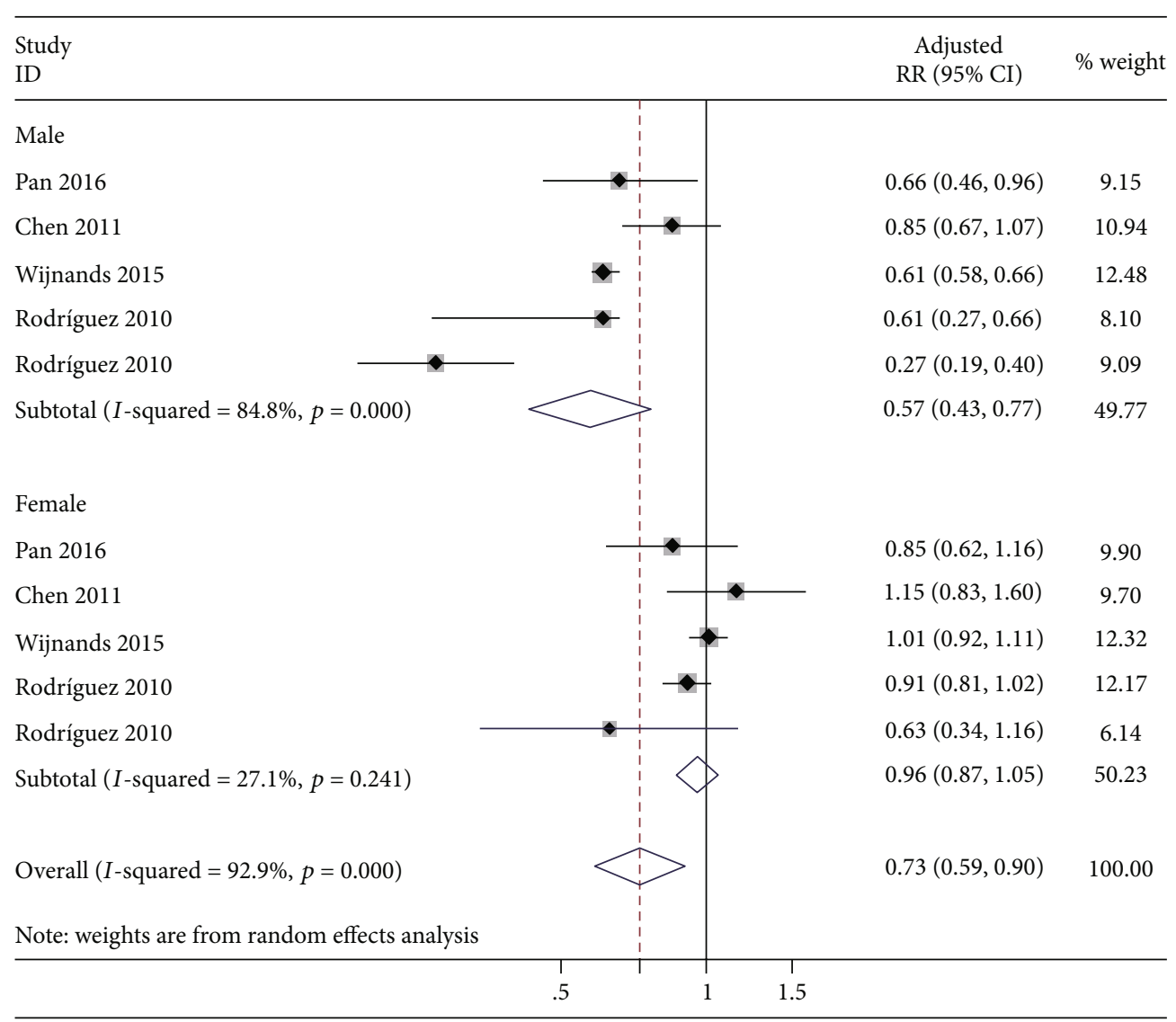

FIgURE 4: Sex-specific analysis of the risk of gout in individuals with DM. RR: relative risk; CI: confidence interval.

of this result were further analyzed. On the one hand, the choice of controls in this study [18] was not rigorous, which only took those without DM in the database as the control group; however, the important factors such as age and gender were not matched with the DM group. On the other hand, some important confounding factors were not adjusted, such as chronic kidney disease, chronic heart failure, and different types of diuretics, which were confirmed to be linked to a significant increase of the risk of gout in previous studies [26-28].

The reverse correlation between DM and the risk of gout may be explained by the uricosuric effect of glycosuria, which generally occurs when serum glucose levels exceed $10 \mathrm{mmol} / \mathrm{L}$ [25]. Some studies indicated that moderately elevated serum glucose levels were related to higher serum uric acid levels, while higher glucose levels sufficient to cause glycosuria $(>10 \mathrm{mmol} / \mathrm{L})$ were correlated with lower serum uric acid levels $[25,29,30]$. Indeed, it was observed that the excretion of uric acid was directly proportional to serum glucose levels once glucose load was enough to lead to glycosuria [25]. Moreover, people with prediabetes had a higher risk of developing gout, while the risk dropped to a lower level once they developed diabetes compared to nondiabetics $[17,31]$. Similarly, several studies consistently have shown that people with DM have lower serum uric acid levels than normal individuals $[29,30,32]$. Therefore, glycosuria may increase the excretion of uric acid through a high filtration rate and osmotic diuresis, thus reducing serum uric acid level and the risk of gout in DM [25, 33].

Furthermore, an impaired inflammatory response may be another important reason for the lower risk of gout in DM. Gout is a common acute inflammatory arthritis caused by the deposition of monosodium urate crystals in joints. Urate crystals can rapidly trigger an inflammatory reaction by stimulating the synthesis and release of inflammatory mediators and then amplifying and maintaining a severe inflammatory response [34]. However, many inflammatory processes were found to be damaged in DM, which exactly hindered the intense inflammatory process caused by urate crystals [34]. The impaired inflammatory response found in DM included inhibition of leukocyte chemotaxis and increased leukocyte apoptosis $[35,36]$, decreased response of endothelial cells to permeability factors such as histamine and bradykinin [37], reduced mast cell degranulation [38], damaged adhesion of neutrophils to endothelial cells and migration to inflammatory sites $[39,40]$, and reduced release of cytokines and prostaglandin by neutrophils [41, 42]. These findings further provide a potential biological mechanism and may be the basis of the reverse association observed in this study.

In this meta-analysis, patients with high HbA1c levels or T1DM had a significantly reduced risk of gout, with possible mechanistic explanations such as a poorly controlled or longlasting DM leading to a significantly uricosuric effect of 


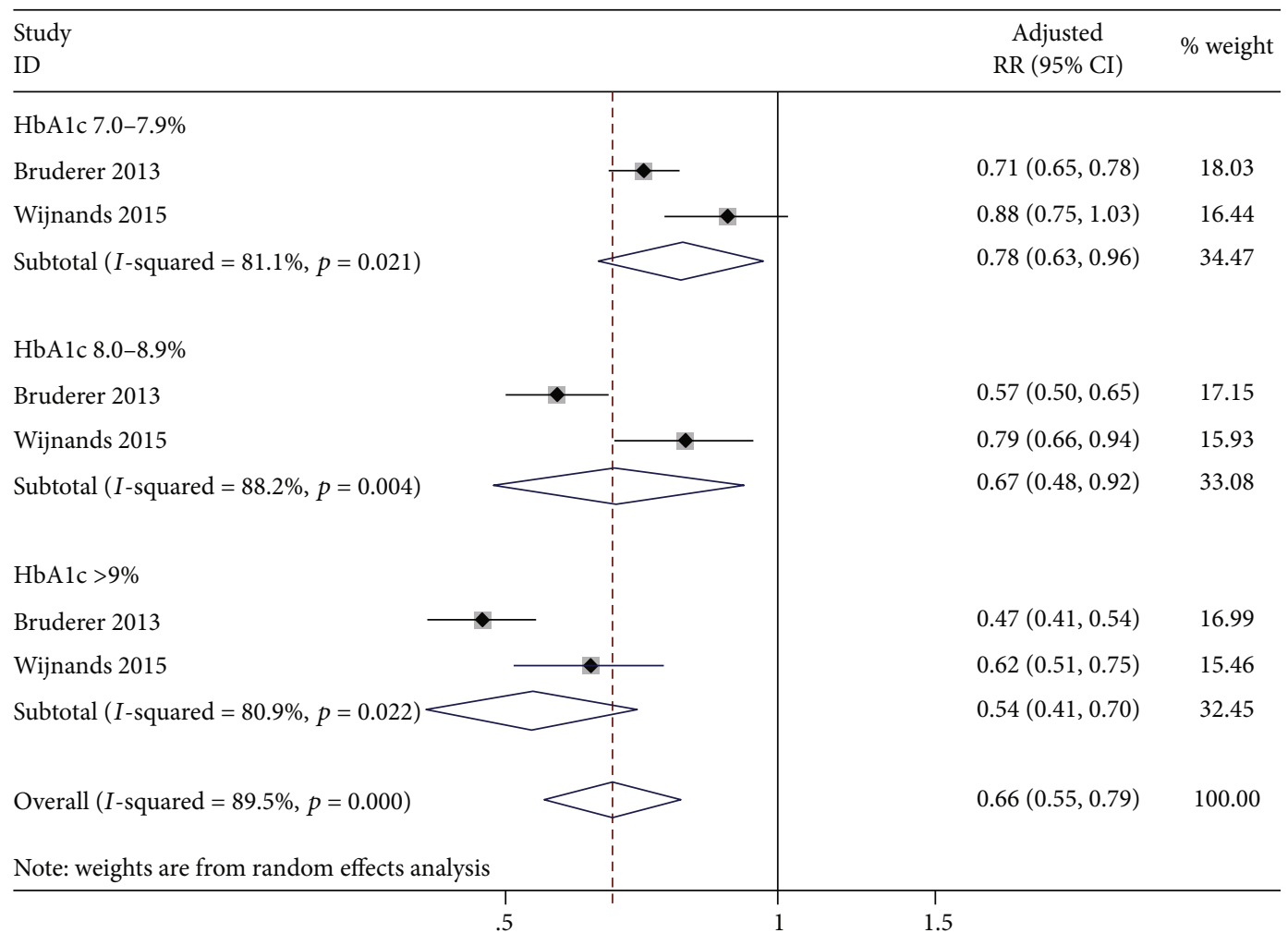

FIgURE 5: Forest plot of association between HbA1c level and the risk of gout. HbA1c: glycated hemoglobin; RR: relative risk; CI: confidence interval.

glycosuria and a seriously impaired inflammatory response. As for the protective association observed only in males rather than females, the sex difference between serum uric acid levels and serum insulin levels may be a reasonable explanation. On the one hand, studies have shown that serum uric acid levels are independently and closely related to the degree of insulin resistance [43-45], and this correlation was significantly stronger among women than men [45]. Besides, since fasting serum insulin levels were significantly higher in both premenopausal and postmenopausal women with hyperuricemia than in men with hyperuricemia [45], sex hormones may also play a role. On the other hand, many studies have found that increased insulin concentration significantly promoted uric acid reabsorption via regulating urate transporter 1 (URAT1) and ATP-binding cassette subfamily G member 2 (ABCG2) in the kidney, thereby reducing urinary excretion of uric acid and increasing serum uric acid levels [43, 46-48]. Therefore, we hypothesize that the protective association in males is likely to be the residual association after cancelling out the effect of insulin on the reabsorption of uric acid. Whereas the effect of insulin on the reabsorption of uric acid was stronger in women than in men, we propose that the opposed effects just cancel each other out in females. Consistent with this gender difference, many evidences have confirmed that serum uric acid levels are predictive of coronary artery disease among women but not among men [49-51], and the sex differences between serum uric acid levels and serum insulin levels may be part of the reason for this difference in correlation [44]. However, it seems impossible to explain this gender difference of T1DM by the effect of insulin on the reabsorption of uric acid. Therefore, there may be other potential mechanisms for gender differences, which obviously need more studies to further confirm their existence.

Nevertheless, this study had some limitations. Firstly, all included studies were conducted using medical registration databases. The definitions of DM and gout were based on diagnostic codes or self-report, and it was inevitable to have some degree of misclassification. Such misclassification could also occur in the selection of the controls. For example, in the study by Wijnands et al. [23], the controls were individuals without a noninsulin antidiabetic drug or an insulin prescription during the whole study period, which might include a few individuals with DM.

Secondly, this was a meta-analysis of observational studies in which several potential confounding factors were adjusted to reduce confounding bias. However, due to some objective reasons of data collection, the confounding factors adjusted for each study were different and some unadjusted confounding factors in the original studies could not be ignored. Some comorbidities (such as hypertension, hyperlipidemia, congestive heart failure, and chronic kidney disease) $[2,28]$ and comedications (such as statins, low-dose aspirin, and especially different types of diuretics) $[2,27,52]$ have been shown to be associated with a significantly increased risk of gout in previous studies. However, except for the study by Wijnands et al. [23] which adjusted both comorbidities and comedications as confounding factors, more comorbidities 
were adjusted but comedications were ignored in other studies. In addition, other important risk factors for gout, such as dietary exposure and physical activity, were not adjusted in most studies. Fortunately, all studies were adjusted by BMI associated with dietary habits and physical activity to control these risk factors to a certain extent. Moreover, the effects of antidiabetic drugs and uric acid-lowering drugs on the results also needed to be considered. Bruderer et al. [53] provided evidence that different types of antidiabetic drugs did not alter the risk of gout, while the use of urate-lowering drugs to treat hyperuricemia can affect the risk of gout in theory, and individuals with $\mathrm{DM}$ are generally more likely to treat hyperuricemia than nondiabetic individuals, as diabetic patients have more chance to discover hyperuricemia during the follow-up of the disease. Unfortunately, none of the included studies provided data on the use of uric acid-lowering drugs. Therefore, these results should be interpreted with caution, and more high-quality prospective cohort studies are needed in the future to provide more substantial evidence.

Thirdly, although we had tried our best to collect all available data, this meta-analysis included fewer original studies and had some degree of heterogeneity, even if we exerted our utmost effort to find the source of heterogeneity. Therefore, more prospective cohort studies are required in the future to confirm the results and more basic studies are needed to explore potential molecular mechanisms.

\section{Conclusion}

In conclusion, this meta-analysis suggested that DM reduced the future risk of gout and the protective effect was stronger in males, T1DM, or DM with a high HbAlc level. The substantial role of the uricosuric effect of glycosuria and the impaired inflammatory response might offer potential mechanisms. These findings may appear counterintuitive, but it is not contradictory to emphasize the prevention of DM and gout simultaneously. Gout should be prevented by dietary adjustments or treating hyperuricemia rather than by focusing on DM. Diabetes professionals should be aware of the relationship between DM and gout, especially in patients with well-controlled DM. These evidences might even change the treatment strategy of diabetic patients to use uric acid-lowering drugs more aggressively.

\section{Conflicts of Interest}

The authors declare that they have no conflicts of interest.

\section{Authors' Contributions}

Huijian Ma and Xiaoli Li conceptualised this study. Xiaoli Li and Yuling Xing searched and screened the documents. Xiaoli Li, Lianju Li, and Tiantian Cheng completed data extraction and quality assessment. Xiaoli Li and Shaohui Ren conducted statistical analysis and made interpretations for the results. Xiaoli Li wrote the first draft of the manuscript. Huijuan Ma reviewed and revised the manuscript. All authors have read, revised, and reviewed the final manuscript.

\section{Acknowledgments}

This study was supported by Natural Science Foundation of Hebei Province (No. H2019307108).

\section{Supplementary Materials}

Supplementary 1. Table S1. Sensitivity analysis of the risk of gout in DM patients and controls.

Supplementary 2. Excel S1-S2. The retrieval history in PubMed and EMBASE databases.

\section{References}

[1] E. Roddy and M. Doherty, "Epidemiology of gout," Arthritis Research \& Therapy, vol. 12, no. 6, p. 223, 2010.

[2] C. F. Kuo, M. J. Grainge, W. Zhang, and M. Doherty, "Global epidemiology of gout: prevalence, incidence and risk factors," Nature Reviews Rheumatology, vol. 11, no. 11, pp. 649-662, 2015.

[3] G. Hu, P. Jousilahti, and J. Tuomilehto, "Joint effects of history of hypertension at baseline and type 2 diabetes at baseline and during follow-up on the risk of coronary heart disease," European Heart Journal, vol. 28, no. 24, pp. 3059-3066, 2007.

[4] M. E. Pavkov, W. C. Knowler, K. V. Lemley, C. C. Mason, B. D. Myers, and R. G. Nelson, "Early renal function decline in type 2 diabetes," Clinical Journal of the American Society of Nephrology, vol. 7, no. 1, pp. 78-84, 2012.

[5] E. Krishnan, "Reduced glomerular function and prevalence of gout: NHANES 2009-10," PLoS One, vol. 7, no. 11, article e50046, 2012.

[6] M. A. McAdams-DeMarco, J. W. Maynard, A. N. Baer, and J. Coresh, "Hypertension and the risk of incident gout in a population-based study: the Atherosclerosis Risk In Communities cohort," The Journal of Clinical Hypertension, vol. 14, no. 10, pp. 675-679, 2012.

[7] M. Doherty, T. L. Jansen, G. Nuki et al., "Gout: why is this curable disease so seldom cured?," Annals of the Rheumatic Diseases, vol. 71, no. 11, pp. 1765-1770, 2012.

[8] H. K. Choi, M. A. De Vera, and E. Krishnan, "Gout and the risk of type 2 diabetes among men with a high cardiovascular risk profile," Rheumatology, vol. 47, no. 10, pp. 1567-1570, 2008.

[9] S. C. Kim, J. Liu, and D. H. Solomon, "Risk of incident diabetes in patients with gout: a cohort study," Arthritis \& Rheumatology, vol. 67, no. 1, pp. 273-280, 2015.

[10] Y. H. Rho, N. Lu, C. E. Peloquin et al., "Independent impact of gout on the risk of diabetes mellitus among women and men: a population-based, BMI-matched cohort study," Annals of the Rheumatic Diseases, vol. 75, no. 1, pp. 91-95, 2015.

[11] A. Pan, G. G. Teng, J.-M. Yuan, and W.-P. Koh, "Bidirectional association between diabetes and gout: the Singapore Chinese Health Study," Scientific Reports, vol. 6, no. 1, p. 25766, 2016.

[12] S. Kodama, K. Saito, Y. Yachi et al., "Association between serum uric acid and development of type 2 diabetes," Diabetes Care, vol. 32, no. 9, pp. 1737-1742, 2009.

[13] R. Suppiah, A. Dissanayake, and N. Dalbeth, "High prevalence of gout in patients with type 2 diabetes: male sex, renal impairment, and diuretic use are major risk factors," The New Zealand Medical Journal, vol. 121, no. 1283, pp. 43-50, 2008. 
[14] S.-Y. Chen, C.-L. Chen, and M.-L. Shen, "Manifestations of metabolic syndrome associated with male gout in different age strata," Clinical Rheumatology, vol. 26, no. 9, pp. 14531457, 2007.

[15] S. Y. Chen, C. L. Chen, M. L. Shen, and N. Kamatani, "Trends in the manifestations of gout in Taiwan," Rheumatology (Oxford), vol. 42, no. 12, pp. 1529-1533, 2003.

[16] I. Anagnostopoulos, E. Zinzaras, I. Alexiou et al., "The prevalence of rheumatic diseases in central Greece: a population survey," BMC Musculoskeletal Disorders, vol. 11, no. 1, p. 98, 2010.

[17] G. Rodríguez, L. C. Soriano, and H. K. Choi, "Impact of diabetes against the future risk of developing gout," Annals of the Rheumatic Diseases, vol. 69, no. 12, pp. 2090-2094, 2010.

[18] J.-H. Chen, W.-T. Yeh, S.-Y. Chuang, Y.-Y. Wu, and W.-H. Pan, "Gender-specific risk factors for incident gout: a prospective cohort study," Clinical Rheumatology, vol. 31, no. 2, pp. 239245, 2011.

[19] A. Stang, "Critical evaluation of the Newcastle-Ottawa scale for the assessment of the quality of nonrandomized studies in meta-analyses," European Journal of Epidemiology, vol. 25, no. 9, pp. 603-605, 2010.

[20] A. Arab, N. Rafie, M. Mansourian, M. Miraghajani, and H. Hajianfar, "Dietary patterns and semen quality: a systematic review and meta-analysis of observational studies," Andrology, vol. 6, no. 1, pp. 20-28, 2018.

[21] J. Zhang and K. F. Yu, "What's the relative risk? A method of correcting the odds ratio in cohort studies of common outcomes," JAMA, vol. 280, no. 19, pp. 1690-1691, 1998.

[22] J. P. Higgins, S. G. Thompson, J. J. Deeks, and D. G. Altman, "Measuring inconsistency in meta-analyses," BMJ, vol. 327, no. 7414, pp. 557-560, 2003.

[23] J. M. Wijnands, C. M. van Durme, J. H. Driessen et al., "Individuals with type 2 diabetes mellitus are at an increased risk of gout but this is not due to diabetes: a population-based cohort study," Medicine, vol. 30, no. 32, article e1358, 2015.

[24] S. G. Bruderer, M. Bodmer, S. S. Jick, and C. R. Meier, "Diabetes and the risk of incident gout," Pharmacoepidemiology and Drug Safety, vol. 22, pp. 46-47, 2013.

[25] D. G. Cook, A. G. Shaper, D. S. Thelle, and T. P. Whitehead, "Serum uric acid, serum glucose and diabetes: relationships in a population study," Postgraduate Medical Journal, vol. 62, no. 733, pp. 1001-1006, 1986.

[26] W. Wang, V. M. Bhole, and E. Krishnan, "Chronic kidney disease as a risk factor for incident gout among men and women: retrospective cohort study using data from the Framingham Heart Study," BMJ Open, vol. 5, no. 4, article e006843, 2015.

[27] S. Bruderer, M. Bodmer, S. S. Jick, and C. R. Meier, "Use of diuretics and risk of incident gout: a population-based casecontrol study," Arthritis \& Rhematology, vol. 66, no. 1, pp. 185-196, 2014.

[28] H. K. Choi, K. Atkinson, E. W. Karlson, and G. Curhan, “Obesity, weight change, hypertension, diuretic use, and risk of gout in men: the health professionals follow-up study," Archives of Internal Medicine, vol. 165, no. 7, pp. 742-748, 2005.

[29] H. K. Choi and E. S. Ford, "Haemoglobin A1c, fasting glucose, serum C-peptide and insulin resistance in relation to serum uric acid levels - the Third National Health and Nutrition Examination Survey," Rheumatology, vol. 47, no. 5, pp. 713717,2008
[30] T. P. Whitehead, I. Jungner, D. Robinson, W. Kolar, A. Pearl, and A. Hale, "Serum urate, serum glucose and diabetes," Annals of Clinical Biochemistry, vol. 29, no. 2, pp. 159-161, 1992.

[31] Q. Liu, G. Gamble, K. Pickering, S. Morton, and N. Dalbeth, "Prevalence and clinical factors associated with gout in patients with diabetes and prediabetes," Rheumatology, vol. 51, no. 4, pp. 757-759, 2012.

[32] F. Wei, B. Chang, X. Yang, Y. Wang, L. Chen, and W.-D. Li, "Serum uric acid levels were dynamically coupled with hemoglobin A1c in the development of type 2 diabetes," Scientific Reports, vol. 6, article 28549, 2016.

[33] R. E. Gilbert, "Sodium-glucose linked transporter-2 inhibitors: potential for renoprotection beyond blood glucose lowering?," Kidney International, vol. 86, no. 4, pp. 693-700, 2014.

[34] H. K. Choi, D. B. Mount, A. M. Reginato, American College of Physicians, and American Physiological Society, "Pathogenesis of gout," Annals of Internal Medicine, vol. 143, no. 7, pp. 499516, 2005.

[35] P. Moriguchi, P. Sannomiya, P. F. Lara, R. M. Oliveira-Filho, K. V. Greco, and L. S. Sudo-Hayashi, "Lymphatic system changes in diabetes mellitus: role of insulin and hyperglycemia," Diabetes/Metabolism Research and Reviews, vol. 21, no. 2, pp. 150-157, 2005.

[36] T. C. Alba-Loureiro, C. D. Munhoz, J. O. Martins et al., "Neutrophil function and metabolism in individuals with diabetes mellitus," Brazilian Journal of Medical and Biological Research, vol. 40, no. 8, pp. 1037-1044, 2007.

[37] Z. B. Fortes, J. G. Lerne, and R. Scivoletto, "Vascular reactivity in diabetes mellitus: role of the endothelial cell," British Journal of Pharmacology, vol. 79, no. 3, pp. 771-781, 1983.

[38] S. C. Cavalher-Machado, W. Tavares de Lima, A. S. Damazo et al., "Down-regulation of mast cell activation and airway reactivity in diabetic rats: role of insulin," The European Respiratory Journal, vol. 24, no. 4, pp. 552-558, 2004.

[39] P. Sannomiya, M. A. A. Pereira, and J. Garcia-Leme, "Inhibition of leukocyte chemotaxis by serum factor in diabetes mellitus: selective depression of cell responses mediated by complement-derived chemoattractants," Agents and Actions, vol. 30, no. 3-4, pp. 369-376, 1990.

[40] Z. B. Fortes, S. P. Farsky, M. A. Oliveira, and J. Garcia-Leme, "Direct vital microscopic study of defective leukocyteendothelial interaction in diabetes mellitus," Diabetes, vol. 40, no. 10, pp. 1267-1273, 1991.

[41] J. de Oliveira Martins, A. R. Meyer-Pflug, T. C. Alba-Loureiro et al., "Modulation of lipopolysaccharide-induced acute lung inflammation: role of insulin," Shock, vol. 25, no. 3, pp. 260266, 2006.

[42] T. C. Alba-Loureiro, E. F. Martins, R. G. Landgraf, S. Jancar, R. Curi, and P. Sannomiya, "Role of insulin on PGE2 generation during LPS-induced lung inflammation in rats," Life Sciences, vol. 78, no. 6, pp. 578-585, 2006.

[43] F. Facchini, Y. D. Chen, C. B. Hollenbeck, and G. M. Reaven, "Relationship between resistance to insulin-mediated glucose uptake, urinary uric acid clearance, and plasma uric acid concentration," JAMA, vol. 266, no. 21, pp. 3008-3011, 1991.

[44] H. Vuorinen-Markkola and H. Yki-Jarvinen, "Hyperuricemia and insulin resistance," The Journal of Clinical Endocrinology and Metabolism, vol. 78, no. 1, pp. 25-29, 1994.

[45] P. Chou, K. C. Lin, H. Y. Lin, and S. T. Tsai, "Gender differences in the relationships of serum uric acid with fasting serum 
insulin and plasma glucose in patients without diabetes," The Journal of Rheumatology, vol. 28, no. 3, pp. 571-576, 2001.

[46] E. W. Holmes, W. N. Kelley, and J. B. Wyngaarden, "The kidney and uric acid excretion in man," Kidney International, vol. 2, no. 3, pp. 115-118, 1972.

[47] F. P. Cappuccio, P. Strazzullo, E. Farinaro, and M. Trevisan, "Uric acid metabolism and tubular sodium handling. Results from a population-based study," JAMA, vol. 270, no. 3, pp. 354-359, 1993.

[48] D. Toyoki, S. Shibata, E. Kuribayashi-Okuma et al., "Insulin stimulates uric acid reabsorption via regulating urate transporter 1 and ATP-binding cassette subfamily G member 2," American Journal of Physiology. Renal Physiology, vol. 313, no. 3, pp. F826-F834, 2017.

[49] J. Fang and M. H. Alderman, "Serum uric acid and cardiovascular mortality. The NHANES I Epidemiologic Follow-up Study, 1971-1992," JAMA, vol. 283, no. 18, pp. 2404-2410, 2000.

[50] B. F. Culleton, M. G. Larson, W. B. Kannel, and D. Levy, "Serum uric acid and risk for cardiovascular disease and death: the Framingham Heart Study," Annals of Internal Medicine, vol. 131, no. 1, pp. 7-13, 1999.

[51] D. S. Freedman, D. F. Williamson, E. W. Gunter, and T. Byers, "Relation of serum uric acid to mortality and ischemic heart disease. The NHANES I Epidemiologic Follow-up Study," American Journal of Epidemiology, vol. 141, no. 7, pp. 637644, 1995.

[52] H. K. Choi, L. C. Soriano, Y. Zhang, and L. A. G. Rodriguez, "Antihypertensive drugs and risk of incident gout among patients with hypertension: population based case-control study," BMJ, vol. 344, article d8190, 2012.

[53] S. G. Bruderer, M. Bodmer, S. S. Jick, and C. R. Meier, "Poorly controlled type 2 diabetes mellitus is associated with a decreased risk of incident gout: a population-based casecontrol study," Annals of the Rheumatic Diseases, vol. 74, no. 9, pp. 1651-1658, 2015. 